\title{
A!
}

This is an electronic reprint of the original article.

This reprint may differ from the original in pagination and typographic detail.

Merikoski, J.; Maunuksela, J.; Myllys, Markko; Timonen, J.; Alava, M. J.

\section{Temporal and spatial persistence of combustion fronts in paper}

\section{Published in:}

Physical Review Letters

DOI:

10.1103/PhysRevLett.90.024501

Published: 17/01/2003

Document Version

Publisher's PDF, also known as Version of record

\section{Please cite the original version:}

Merikoski, J., Maunuksela, J., Myllys, M., Timonen, J., \& Alava, M. J. (2003). Temporal and spatial persistence of combustion fronts in paper. Physical Review Letters, 90(2), 1-4. [024501].

https://doi.org/10.1103/PhysRevLett.90.024501

This material is protected by copyright and other intellectual property rights, and duplication or sale of all or part of any of the repository collections is not permitted, except that material may be duplicated by you for your research use or educational purposes in electronic or print form. You must obtain permission for any other use. Electronic or print copies may not be offered, whether for sale or otherwise to anyone who is not an authorised user. 


\title{
Temporal and Spatial Persistence of Combustion Fronts in Paper
}

\author{
J. Merikoski, J. Maunuksela, M. Myllys, and J. Timonen \\ Department of Physics, University of Jyväskylä, P.O. Box 35, FIN-40351 Jyväskylä, Finland \\ M. J. Alava \\ Laboratory of Physics, Helsinki University of Technology, P.O. Box 1100, FIN-02015 HUT, Espoo, Finland
}

(Received 21 March 2002; published 13 January 2003)

\begin{abstract}
The spatial and temporal persistence, or first-return distributions are measured for slow-combustion fronts in paper. The stationary temporal and (perhaps less convincingly) spatial persistence exponents agree with the predictions based on the front dynamics, which asymptotically belongs to the KardarParisi-Zhang universality class. The stationary short-range and the transient behavior of the fronts are non-Markovian, and the observed persistence properties thus do not agree with the predictions based on Markovian theory. This deviation is a consequence of additional time and length scales, related to the crossovers to the asymptotic coarse-grained behavior.
\end{abstract}

DOI: 10.1103/PhysRevLett.90.024501

PACS numbers: 47.54.+r, 02.50.-r, 05.40.-a, 81.10.Aj

Nonequilibrium systems present a wide range of questions. One can try to define necessary conditions for the existence of a stationary state and then proceed to a characterization of that state. If the dynamics is of an inherently transient character, more complicated scenarios arise. Unlike in the stationary state, in which the behavior of the order parameter is described by some probability distribution, one encounters concepts such as aging and coarsening which reflect the correlations [1].

A particularly concise and topical approach in nonequilibrium dynamics is persistence, often defined as the probability $P(t)$ that, at a point in space, a fluctuating nonequilibrium field (such as a diffusion field) does not change sign up to time $t[2,3]$. This probability may decay algebraically, $P(t) \sim t^{-\theta}$, with a persistence exponent $\theta$. In some cases $\theta$ may be unrelated to any of the other exponents that characterize the system, in particular, those describing the critical decay of the temporal and spatial correlation functions. Of special interest in this respect are non-Markovian random processes, for the extra challenges they present.

Because of the statistical nature of the problem, clear experimental demonstrations of persistence are still quite rare [4-7]. In the present Letter, we consider persistence as observed in the experiments on the propagation of slow-combustion fronts in paper sheets. We have earlier [8-10] shown that at long spatial and temporal scales the dynamics of these fronts follows the universality class of the $(1+1)$-dimensional Kardar-Parisi-Zhang (KPZ) equation $[11,12]$

$$
\frac{\partial h}{\partial t}=\nu \nabla^{2} h+\frac{\lambda}{2}(\nabla h)^{2}+\eta,
$$

where $h=h(x, t)$ is the height of the interface at point $x$ and time $t, \nu$ is the surface tension parameter, $\lambda$ is the strength of the nonlinearity, and $\eta=\eta(x, t)$ is Gaussian white noise. We have also shown $[9,10]$ that at short scales the short-range correlations in the effective noise prevent the system from displaying true scaling behavior.

We consider here the first-return properties of the experimentally observed fronts, $h(x, t)$, in spatial and temporal domains, at both stationary and transient stages, and compare them with various theoretical predictions. For short time and length scales, the correlations in the noise in our experiments are relevant, and the dynamics is non-Markovian. We study the implications of this on persistence.

A complete description of our experimental setup is given in Ref. [10]. We have chosen to analyze data for the $80 \mathrm{gm}^{-2}$ copier paper as a typical case, and also since it presents the best statistics. In Fig. 1 we show the heightfluctuation field observed in a typical sample "burn."

In the absence of nonlinearity $(\lambda=0)$, the long-range dynamics would be described by a linear diffusion equation with thermal noise, the Edwards-Wilkinson (EW) equation. This case was analyzed in the temporal domain by Krug et al. in the context of linear growth equations [13] and by Bray and Majumdar in terms of the spatial return characteristics [14]. In the stationary state one finds $\theta_{s}=1 / 2$, which for these linear equations is the same as for the transient behavior.

For $\lambda \neq 0$ the stochastic process, the KPZ height fluctuations, is non-Gaussian, and the stationary and transient temporal persistences are theoretically difficult to analyze. Kallabis and Krug observed, starting from a numerical growth model, that the persistence behavior can be characterized by considering the scaling functions of the general persistence probability $P\left(t_{0}, t\right)$, where $t$ is measured beginning from time $t_{0}$ after the start of the kinetics from a flat initial profile [15]. This has two limiting behaviors, the transient behavior for $t_{0}$ before saturation and the stationary-state behavior for $t_{0}$ after it, thus defining two persistence exponents. The numerical results [15] follow the general conjecture that for the stationary 


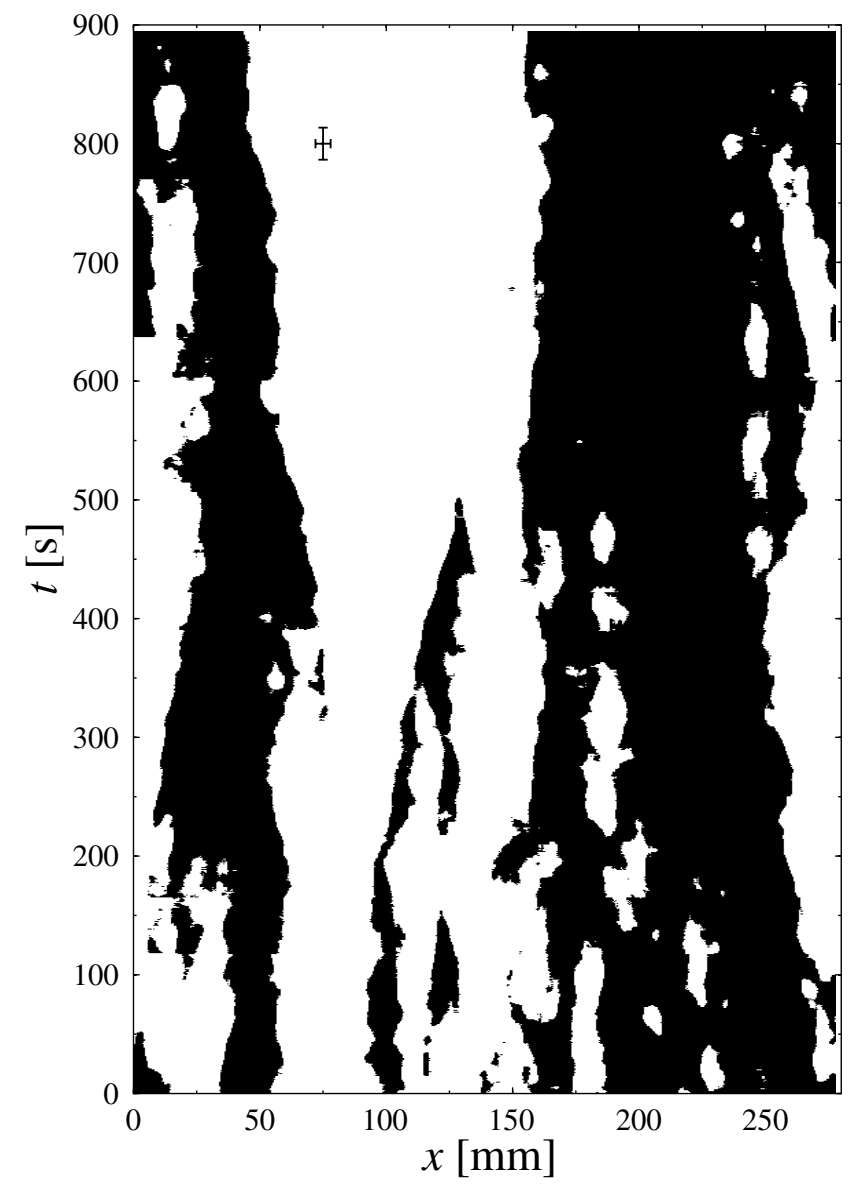

FIG. 1. An example of a combustion experiment. In the height-fluctuation field the black color indicates fluctuations in the positive and white in the negative direction. The crossover scales $\tau_{c}$ and $\ell_{c}$, see Figs. 2(b) and 3(b), are marked by bars.

state

$$
\theta_{s}=1-\beta,
$$

where $\beta$ describes the temporal two-point correlation function of the interface fluctuations (to be defined below), which can be justified by the self-affine nature of the process. For the transient regime, Kallabis and Krug observed, e.g., that persistence depends on the up-down asymmetry of the dynamics, in their model with values 1.2 and 1.6 for the transient temporal persistence exponents for fluctuations in the up and down directions, respectively [15]. In the stationary state the asymmetry was reflected only as different normalizations of the persistence probability for fluctuations in the up and down directions.

One may likewise consider any particular front at a fixed time $t$ and look at the interface profile as a stochastic process as discussed by Bray and Majumdar [14], who studied first-passage properties in space. In analogy with the temporal case, the probability that the interface stays above or below a given reference level may have a powerlaw decay. Moreover, depending on whether the configuration represents the stationary state or whether it has essentially finite fluctuations, one again has two different exponents. In terms of the interface morphology, they can be related to the roughness exponent $\chi$ (see below).

To characterize persistence, we consider $f_{ \pm}^{\text {temp }}(\tau)$, the first-return distributions, i.e., the distributions of return time $\tau$, defined as the time a variable stays above $(+)$ or below $(-)$ a given reference level. The persistence exponents $\theta_{ \pm}^{\text {temp }}$ and $\theta_{ \pm}^{\text {spat }}$ describe the decay of the related temporal and spatial persistence probabilities, respectively, and are defined via $[13,14]$

$$
P_{ \pm}^{\text {temp }}(\tau) \sim \tau^{-\theta_{ \pm}^{\text {temp }}} \quad \text { and } \quad P_{ \pm}^{\text {spat }}(\ell) \sim \ell^{-\theta_{ \pm}^{\text {spat }}},
$$

where $\tau$ and $\ell$ denote the persistent time- and length scales. In the transient regime these quantities are computed by sampling within a suitably chosen time window (see below), and denoted by a tilde, e.g., $\tilde{\theta}_{ \pm}^{\text {temp }}$.

Since a combustion front propagates with a finite average velocity $v$, we look at the fluctuations $\delta h(x, t)$, at a fixed point $x=x_{0}$ and time $t$, in the front $h(x, t)$ around its average height,

$$
\delta h(x, t) \equiv h(x, t)-\bar{h}(t) .
$$

Here the overbar denotes a spatial average at time $t$. For practical purposes we define the return times as follows: $\tau_{+}$is the length of the time interval between $t_{1}$ and $t_{2}$ such that $\delta h\left(x_{0}, t_{1}\right)=0=\delta h\left(x_{0}, t_{2}\right)$, and $\delta h\left(x_{0}, t\right)>0$ for all $t \in] t_{1}, t_{2}\left[\right.$. The return time $\tau_{-}$is defined analogously for $\delta h\left(x_{0}, t\right)<0$. For discrete sampling times we determine the crossing times by using linear interpolation.

The temporal persistence probabilities [13] are related to the corresponding first-return distribution via

$$
P_{ \pm}^{\mathrm{temp}}(\tau) \equiv P\left(\tau_{ \pm} \geq \tau\right)=1-\int_{-\infty}^{\tau} f_{ \pm}^{\mathrm{temp}}\left(\tau^{\prime}\right) d \tau^{\prime} .
$$

As explained in Ref. [16], in discrete time (and also in discrete space) sampling, one misses very short excursions, and correct normalization of $P_{ \pm}^{\text {temp }}$ and $P_{ \pm}^{\text {spat }}$ is difficult. Therefore, we prefer to use the distributions $f_{ \pm}^{\text {temp }}$ and $f_{ \pm}^{\text {spat }}$, instead of their integrals, for the determination of the persistence exponents. In the limit of long time and length scales ( $\tau$ and $\ell$ ), the problems in these functions due to discrete sampling should disappear.

The corresponding spatial quantities [14] at fixed times are defined analogously. $P_{ \pm}^{\text {spat }}(\ell)$ is the probability that the front stays above $(+)$ or below $(-)$ the reference level over a distance $\ell$, and $f_{ \pm}^{\text {spat }}(\ell)$ denotes the corresponding return-length distribution.

The conjecture of Eq. (2) for the temporal persistence, and the arguments of Bray and Majumdar, make it necessary to outline the behavior of the two-point correlation functions. These, and the associated critical exponents are defined in the usual way as [12] 


$$
\begin{aligned}
& C^{\text {temp }}(\tau)=\langle\overline{|\delta h(x, t)-\delta h(x, t+\tau)|}\rangle \sim \tau^{\beta}, \\
& C^{\text {spat }}(\ell)=\langle\overline{|\delta h(x, t)-\delta h(x+\ell, t)|}\rangle \sim \ell^{\chi},
\end{aligned}
$$

in which the overbar denotes spatial and the brackets disorder averaging [17]. In our earlier works, the longrange scaling of the correlation functions was shown to be described by the KPZ asymptotics with $\chi \simeq 1 / 2$ and $\beta \simeq 1 / 3[8-10]$.

In Fig. 2 we show our experimental results for temporal persistence in the stationary state. Above a crossover scale $\tau_{c}$ of the order of $10 \mathrm{sec}$ (corresponding to fronts that have propagated, on the average, by about $45 \mathrm{~mm}$, see Fig. 1 again), this figure indicates agreement with the theoretical expectation, $\theta_{ \pm}^{\text {temp }}=1-\beta$. The dashed line in Fig. 2(a) follows Eq. (2). Below $\tau_{c}$ the data in Fig. 2(a) show no real scaling regime, in analogy to the curvature visible in $C_{ \pm}^{\text {temp }}(\tau)$ shown Fig. 2(b). We find no difference between the first-return distributions in the positive and negative directions since our distributions are separately normalized to one, so the anisotropy does not appear in the plots.

The measured first-return distributions for spatial persistence in the stationary state are shown in Fig. 3. Now the asymptotic behavior is roughly consistent with $\theta_{ \pm}^{\text {spat }}=$ $1-\chi$. The rather long crossover regions [10] in the correlation functions $C(\tau)$ and $C(\ell)$ in Figs. 2(b) and 3(b), which precede the long-range regimes, are directly visible in the persistence data as well. The crossover is here located at about $10 \mathrm{~mm}$, which is less than the scale $\tau_{c} / v$ would indicate.

The expected persistence behavior takes place only on long enough scales, where the physics is coarse grained so as to obey the KPZ equation. There are correlations in the effective noise (local height increments), with decay scales of a few seconds and a few millimeters [9]. In the short-range regime of the stationary-state data, the spatial and temporal statistics are quite far from the scaling
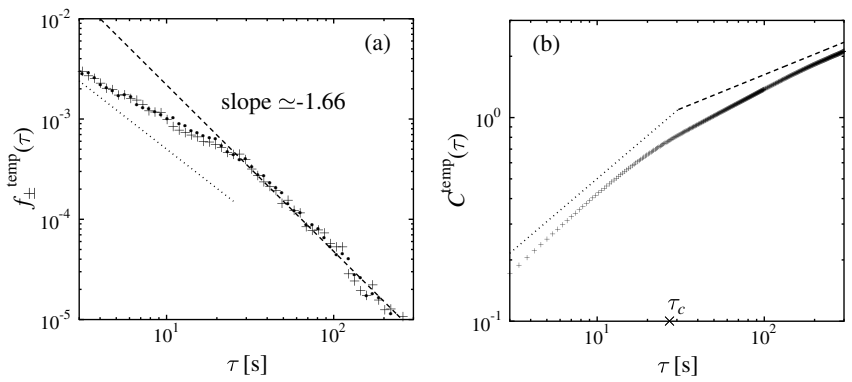

FIG. 2. The stationary temporal (a) first-return distributions $f_{+}^{\text {temp }}(\tau)$ and $f_{-}^{\text {temp }}(\tau)$ denoted by crosses and dots, respectively, and (b) correlation function $C^{\mathrm{temp}}(\tau)$. In all figures, the distributions are normalized to unity and the horizontal axis is logarithmically binned. The dashed and the dotted lines correspond to the asymptotic KPZ and apparent short-range values [10] of $\beta$, in (a) via the conjecture $f(\tau) \sim \tau^{-(2-\beta)}$ from Eq. (2). conjecture " $\theta=1-\beta$." This agrees with the fact that the dynamics becomes Markovian only asymptotically. The short-range persistence does not result from an effectively stationary process that would differ from the long-range dynamics only by the fact that the twopoint exponents are not defined. The deviation is greatest in the case of the temporal behavior, for which persistence decays slower than expected from the correlation function.

The first-return distributions for the transient-time regime of the dynamics are shown in Fig. 4 for a time window from the beginning of the burn to just below the saturation time. In the temporal case the simulation result for the model of Ref. [15] was $\tilde{\theta}_{ \pm}^{\text {temp }} \sim 1.2, \ldots, 1.6$. In our temporal transient distribution shown in Fig. 4(a), the asymptotics beyond the crossover from short-range behavior shows a steeper decay than in the saturated distribution, but the length of this asymptotic part is so short that definitive conclusions cannot be made. It may as well be related to some kind of cutoff behavior. No indications of an up-down anisotropy can be seen. In the spatial transient distribution the asymptotics is better defined, and it is interesting to notice that it is given by $\ell^{-(2-\chi)}$ with $\chi=1 / 2$ as in the saturated regime [see Fig. 4(b)].

The main features of the observed transient persistences can be summarized in two findings: First, in our data the spatial long-range scaling is reminiscent of the stationary state. Second, there is no simple short-range behavior below the crossover scales. In some cases the short-range scalings resemble power-law ones, albeit over rather short ranges, but the effective persistence exponents are never in agreement with those related to the decay of correlations.

In principle, for a time window above the crossover time $\tau_{c}$ but below the saturation time some "expected" (KPZ) temporal transient behavior could be observed. However, the typically wide [10] crossover region around $\tau_{c}$ would interfere with it, unless the saturation time could be made long enough by, e.g., considerably increasing the
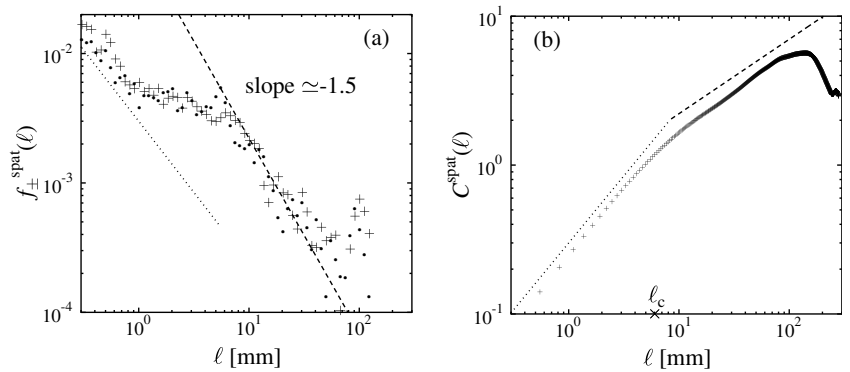

FIG. 3. The stationary spatial (a) first-return distributions $f_{ \pm}^{\text {spat }}(\ell)$ denoted by crosses and dots, respectively, and (b) correlation function $C^{\text {spat }}(\ell)$. The dashed and the dotted lines correspond to the asymptotic KPZ and apparent short-range values [10] of $\chi$, in (a) via the conjecture $f(\ell) \sim \ell^{-(2-\chi)}$ from Eq. (2). 

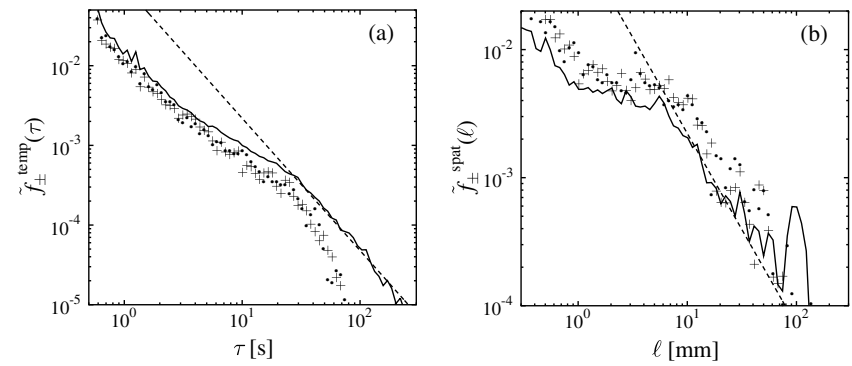

FIG. 4. Transient regime (a) temporal first-return distributions $\tilde{f}_{ \pm}^{\text {temp }}(\tau)$ and (b) spatial first-return distributions $\tilde{f}_{ \pm}^{\text {spat }}(\ell)$. In both cases crosses denote excursions to positive and dots to negative direction. By the full curves we show the averages (over \pm directions) of the stationary distributions $f_{ \pm}^{\text {temp }}$ and $f_{ \pm}^{\text {spat }}$ of Figs. 2 and 3, respectively.

system size. Recall that the origin of the temporal transient exponents in KPZ-type dynamics, as seen in Ref. [15], is the asymmetric interface (valleys and hilltops). In our case, these features can be visible only for $\tau>\tau_{c}$ and $\ell>\ell_{c}[10]$.

To summarize, we have studied the persistence properties of fluctuating combustion fronts in paper. Asymptotically both temporal and spatial persistence follow the theoretical expectations for the stationary case. For the short-range behaviors the KPZ physics is irrelevant, and the first-return distributions deviate from those based on a stationary Markovian stochastic process [18]. Instead, the physics indicates the presence of memory effects. Our results have theoretical implications also for problems, where the KPZ scaling is seen only asymptotically [19].

We wish to acknowledge discussions on stochastic dynamics with Otto Pulkkinen and on kinetic roughening with Tapio Ala-Nissila. This work has been in part supported by the Academy of Finland under the Center of Excellence Program (Project No. 44875).

[1] A. J. Bray, Adv. Phys. 43, 357 (1994).

[2] S. N. Majumdar, Curr. Sci. 77, 370 (1999).

[3] S. N. Majumdar et al., Phys. Rev. Lett. 77, 3704-3707 (1996).

[4] G. P. Wong et al., Phys. Rev. Lett. 86, 4156 (2001).

[5] B. Yurke et al., Phys. Rev. E 56, R40 (1997).

[6] W. Y. Tam et al., Phys. Rev. Lett. 78, 1588 (1997); 79, 957 (1997).

[7] D. B. Dougherty et al., Phys. Rev. Lett. 89, 136102 (2002).

[8] J. Maunuksela et al., Phys. Rev. Lett. 79, 1515 (1997); 80, 5707 (1998).

[9] M. Myllys et al., Phys. Rev. Lett. 84, 1946 (2000).

[10] M. Myllys et al., Phys. Rev. E 64, 036101 (2001).

[11] M. Kardar et al., Phys. Rev. Lett. 56, 889 (1986).

[12] T. Halpin-Healy and Y.-C. Zhang, Phys. Rep. 254, 215 (1995).

[13] J. Krug et al., Phys. Rev. E 56, 2702 (1997).

[14] S. N. Majumdar and A. J. Bray, Phys. Rev. Lett. 86, 3700 (2001).

[15] H. Kallabis and J. Krug, Europhys. Lett. 45, 20 (1999).

[16] S. N. Majumdar et al., Phys. Rev. E 64, 015101 (R) (2001).

[17] For the sake of comparing with the first-return distributions one has to use the "bare" correlation functions. That is, the intrinsic widths cannot be subtracted [10].

[18] I. V. L. Costa et al., cond-mat/0207718.

[19] K. Sneppen et al., Phys. Rev. A 46, R7351 (1992); P. Manneville and H. Chate, Physica D (Amsterdam) 96, 30 (1996); R. Cuerno and M. Castro, Phys. Rev. Lett. 87, 236103 (2001). 